\title{
INTERNAÇÕES EM UNIDADE CRITICA NEONATAL DE UM HOSPITAL INFANTIL PÚBLICO dO PARANÁ
}

\section{Hospitalizations in a neonatal critical unit of a public children's hospital in the state of Paraná}

Elaine Priscila Pechepiura ${ }^{1}$, Michelle Thais Migoto², Fernanda Gabriela Leandro Schaedler ${ }^{3}$, Márcia Helena de Souza Freire ${ }^{4}$

\begin{abstract}
1. Universidade Federal do Paraná (UFPR). ORCID: https://orcid.org/0000-0002-5431-0384.
2. Universidade Federal do Paraná (UFPR). ORCID: https://orcid.org/0000-0002-8546-8694.

3. Universidade Federal do Paraná (UFPR). ORCID: https://orcid.org/0000-0001-6031-8000.

4. Universidade de São Paulo (USP). ORCID: https://orcid.org/0000-0003-3941-3673.
\end{abstract}

CONTATO: Elaine Priscila Pechepiura | Endereço: Rua Ademar de Barros, 715 | Jardim Social | Campo Largo - PR | Telefone: 41998018810 | E-mail: elainepechepiura7@gmail.com

COMO CITAR: Pechepiura EP, Migoto MT, Schaedler FGL, Freire MH de S. Internações em unidade crítica neonatal de um hospital infantil público do Paraná. R. Saúde Públ. Paraná. 2019 Dez;2(2):59-68.

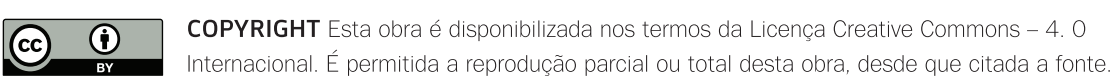

RESUMO A caracterização dos pacientes atendidos em unidades de terapia intensiva neonatal contribui para a gestão dos leitos e o planejamento do cuidado neonatal com impacto na melhoria do estado de saúde e na redução da mortalidade infantil. O objetivo foi caracterizar o perfil dos neonatos internados na Unidade de Terapia Intensiva, em hospital infantil público do Paraná. Estudo transversal quantitativo, com dados de 2018. Verificaram-se 235 internações; $55,7 \%$ do sexo masculino; $46,0 \%$ no período neonatal precoce; 54,0\% nasceram de parto vaginal, 
59,5\% prematuros; 54,8\% apresentaram baixo peso ao nascer; 59,1\% das mães eram procedentes da Regional de Saúde Metropolitana; a mediana do tempo de internação foi de 19 dias. A principal condição de internação foi a prematuridade $(13,1 \%)$, como desfecho prevalente a alta para enfermaria (86,0\%); cinco óbitos (2,1\%). 0 problema de saúde pública dos países em desenvolvimento constituído pelo nascimento prematuro é reiterado, e reforça a necessidade de serviços qualificados.

PALAVRAS-ChAVE: Recém-nascido Prematuro. Unidades de Terapia Intensiva Neonatal. Enfermagem Neonatal. Mortalidade Infantil.

ABSTRACT The characterization of patients assisted in neonatal intensive care units contributes to bed management and neonatal care planning, improving health conditions and reducing child mortality. The objective was to characterize the profile of infants admitted to the Intensive Care Unit of a public children's hospital in the state of Paraná. This is a quantitative, cross-sectional study using data from 2018. There were 235 hospitalizations in $2018 ; 55.7 \%$ were male; $46.0 \%$ in the early neonatal period; $54.0 \%$ were born through vaginal delivery, $59.5 \%$ were premature; $54.8 \%$ had low weight; $59.1 \%$ of the mothers were from the Metropolitan Region Unit. The hospitalization period median was 19 days; prematurity was the main cause of hospitalization (13.1\%). The discharge to other nursery was the main outcome (86.0\%), there were five deaths $(2.1 \%)$. The public health problem in developing countries of premature birth is reiterated and reinforces the need for qualified services.

KEYWORDS: Infant, Premature. Intensive Care Units, Neonatal. Neonatal Nursing. Infant Mortality.

\section{INTRODUÇÃo}

stima-se que em todo o mundo, anualmente, nasçam 15 milhões de recém-nascidos (RN) prematuros. Embora mais de 60,0\% dos nascimentos prematuros ocorram na África e no sul da Ásia, a prematuridade se constitui como um problema global, pois é a principal causa associada à mortalidade de crianças menores de cinco anos de idade ${ }^{1}$.

Os RN prematuros são aqueles que nasceram antes de completarem 37 semanas de idade gestacional (IG), a termo entre 37 e 41 semanas e pós-termo com 42 ou mais semanas de idade gestacional. Sendo que o período neonatal compreende o tempo desde o nascimento até o $28^{\circ}$ dia incompleto de vida. Para os nascimentos pré-termos identificam-se as seguintes subcategorias: prematuro extremo, menos de 28 semanas de IG; muito prematuro, 28 a 32 semanas de IG; prematuro moderado ou tardio, de 32 a 37 semanas de IG ${ }^{1-2}$. Evidências científicas apontam maior vulnerabilidade do RN de menor IG, associado ao muito baixo peso ${ }^{3}$. 
Neste cenário, políticas de atenção à saúde que visam melhorias nas condições de vida e de atenção à saúde de mães e crianças são continuamente traçadas com o objetivo de reduzir a mortalidade infantil4. Sobretudo, a mortalidade neonatal precoce (até o sétimo dia de vida) que responde a 50\% das mortes infantis no Brasil e reflete as condições socioeconômicas e de saúde materna, assim como, a qualidade da atenção prestada no pré-natal, parto, nascimento e ao atendimento neonatal ${ }^{3}$. Entretanto, o acompanhamento prénatal em momento oportuno, com assistência qualificada e humanizada integrando todos os niveis de atenção, responderia às necessidades da gestante e do $\mathrm{RN}^{5}$.

Ainda se considera que no período neonatal há necessidade de cuidados especiais, com atuação tecnicamente qualificada, integral e em tempo oportuno'. Destarte, para o atendimento de neonatos de alto risco é necessária a disponibilidade de leitos críticos, em Unidade de Terapia Intensiva Neonatal (UTIN). Os RN admitidos na UTIN, via de regra, atendem a um ou mais dos seguintes critérios: asfixia grave ao nascer (Apgar < 7 no $5^{\circ}$ minuto); RN pré-termo com peso ao nascer <2.000g; RN <35 semanas de idade gestacional; e RN com outras doenças graves ${ }^{5}$.

Assim, o crescente número de nascimentos prematuros que acarretam expressiva representatividade entre as causas de mortes infantis, relacionado ao aumento proporcional da mortalidade neonatal precoce, reforça a importância de produção de evidências científicas frente a assistência neonatal. Conhecer as características da demanda de internação de prematuros contribuirá para a melhoria do planejamento do cuidado, bem como para a gestão dos leitos neonatais, e, consequentemente, contribui para a redução da morbimortalidade infantil.

Portanto, o objetivo deste estudo foi caracterizar as internações ocorridas na Unidade de Terapia Intensiva Neonatal, de um hospital infantil público da região Sul do Brasil, Paraná.

\section{METODOLOGIA}

Trata-se de um estudo transversal, o qual não permite identificar inferências causais e possiveis vieses de informação, com abordagem quantitativa, realizado na UTIN de um hospital infantil público. Este, como uma unidade própria da Secretaria de Saúde do Estado do Paraná, localiza-se em um município da região metropolitana de Curitiba, e foi inaugurado em 2009. Oferece atendimento especializado de média e alta complexidade às crianças e adolescentes como referência para todo o estado, segundo a lógica do projeto de regionalização da saúde pública. Conta atualmente com 74 leitos ativos, sendo 20 de UTIN, 10 de Unidade de Terapia Intensiva Pediátrica, 24 de Clínica Cirúrgica e 20 de Clínica Médica 5 .

A população de estudo foi indiretamente todos os neonatos que foram internados na UTIN no ano em questão. A coleta de dados secundários se desenvolveu a partir do Sistema de Gestão da Assistência de Saúde do SUS (GSUS), e foram utilizados os Resumos de Alta referentes às internações ocorridas no ano de 2018. Embora os Sistemas de Informação em Saúde (SIS) constituam relevantes fontes de dados secundários com ampla cobertura populacional, a qualidade dos dados registrados deve ser observada, para que representem a realidade estudada?.

Para a organização e análise dos dados foi desenvolvida uma planilha no Excel da Microsoft $®$ com as seguintes variáveis: sexo; idade de internação; via de nascimento (tipo de parto); idade gestacional; peso ao nascer; procedência materna segundo as Regionais de Saúde do Paraná; tempo e desfecho da internação; e diagnósticos médicos segundo a Classificação Estatística Internacional 
de Doenças e Problemas Relacionados à Saúde CID-10. A coleta dos dados ocorreu de fevereiro a abril de 2019, concomitante à análise descritiva utilizando-se de frequências, proporções, médias e medianas, apresentadas em tabelas síntese.

Para alinhamento dos dados, durante o preenchimento da planilha alguns critérios foram adotados como a exclusão de diagnósticos inconclusivos, não confirmados durante a internação. Enquanto as situações clínicas não esclarecidas foram mantidas, destas foram trabalhados 14 diagnósticos sintomáticos, como exemplo: petéquias, vômitos, febre e cianose.

No caso das reinternações de um mesmo neonato, os diagnósticos que se repetiam em relação à primeira internação não foram contabilizados novamente. Quanto ao peso de nascimento, se não informado, foi considerado o peso de admissão na unidade sempre que o neonato tivesse sido admitido no mesmo dia de seu nascimento.

Quanto à idade gestacional, na falta de informações, para recém-nascidos termo foram consideradas 39 semanas, e quando descrito "meia" $(0,5)$ semana contabilizaram-se três dias à idade gestacional.

Em relação ao tempo de internação, frente à divergência entre os dados fornecidos pelo GSUS e dos Resumos de Alta, optou-se pela utilização dos dados presentes no GSUS. Os diagnósticos descritos nos Resumos de Alta foram todos categorizados segundo os capítulos da CID-10.

Com vistas à qualidade, esta pesquisa foi estruturada segundo as recomendações do checklist STROBE - Strengthening the Reporting of Observational Studies in Epidemiology, que contém 22 itens, com indicações do que se deve incluir para uma descrição mais precisa de estudos observacionais $^{8}$
Este estudo integra um dos objetivos de um projeto temático desenvolvido no hospital, e foi aprovado com pareceres de dois Comitês de Ética em Saúde, a saber: do Setor de Ciências da Saúde da UFPR - no 1.618.264, de 01 de julho de 2016; e Hospital do Trabalhador/SES/PR - n 1.698.784. de 25 de agosto de 2016. Por tratar-se de estudo quantitativo com dados secundários foi dispensado o Termo de Consentimento Livre e Esclarecido.

\section{RESULTADOS}

A UTIN em 2018 apresentou taxa de ocupação de 94,58\%. Verificaram-se 223 internações e 12 reinternações; com prevalência do sexo masculino (55,7\%); período neonatal precoce (46,0\%); nascidos de parto vaginal (54,0\%); prematuros (59,5\%) e com peso ao nascer menor de $2.500 \mathrm{~g}$ $(54,8 \%)$. As idades gestacionais variaram de 23 a 41 semanas e dois dias, com média e mediana de 33 semanas. O peso ao nascer variou de $490 \mathrm{~g}$ a $5.050 \mathrm{~g}$, com média de $2.171 \mathrm{~g}$ e mediana de $2.091 \mathrm{~g}$ (TABELA 1)

A idade de internação, que corresponde ao período do nascimento até o dia da internação, divergiu de zero a 185 dias, com média de 23 dias e mediana de nove dias. O tempo de internação variou de horas (menos de um dia) a 146 dias, com média de 28 dias e mediana de 19 dias. 0 desfecho prevalente foi a alta para a enfermaria $(86,0 \%)$ e ocorreram cinco $(2,1 \%)$ óbitos no período (TABELA 1).

A Tabela 2 apresenta o município de residência da mãe do neonato internado na UTIN, segundo as Regionais de Saúde do Paraná, com destaque para

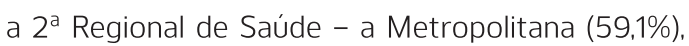
bem como o município de Curitiba que contabilizou 21,7\% das internações do estudo. Houve apenas uma internação de procedência de outro estado. 
Tabela 1 Distribuição (n e \%) dos neonatos segundo variáveis selecionadas, Unidade de Terapia Intensiva Neonatal, Hospital Infantil, Campo Largo, Paraná, 2018

\begin{tabular}{|c|c|c|c|c|}
\hline VARIÁVEL & \multicolumn{2}{|c|}{ CATEGORIA } & NÚMERO (n) & PROPORÇÃO (\%) \\
\hline \multirow[t]{2}{*}{ Sexo } & \multicolumn{2}{|l|}{ Feminino } & 104 & $44,3 \%$ \\
\hline & \multicolumn{2}{|l|}{ Masculino } & 131 & $55,7 \%$ \\
\hline \multirow{3}{*}{ Período (dias) } & \multicolumn{2}{|c|}{ Neonatal precoce } & 108 & $46,0 \%$ \\
\hline & \multicolumn{2}{|c|}{ Neonatal tardio } & 68 & $28,9 \%$ \\
\hline & \multicolumn{2}{|c|}{ Pós-neonatal } & 59 & $25,1 \%$ \\
\hline \multirow{3}{*}{ Via de nascimento } & \multicolumn{2}{|l|}{ Cesariana } & 89 & $37,9 \%$ \\
\hline & \multicolumn{2}{|l|}{ Vaginal } & 127 & $54,0 \%$ \\
\hline & \multicolumn{2}{|c|}{ Não informado } & 19 & $8,1 \%$ \\
\hline \multirow{6}{*}{$\begin{array}{l}\text { Idade gestacional } \\
\text { (semanas) }\end{array}$} & \multirow{3}{*}{ Pré-termo } & Prematuro extremo & 41 & $17,4 \%$ \\
\hline & & Muito prematuro & 44 & $18,7 \%$ \\
\hline & & Prematuro moderado ou tardio & 55 & $23,4 \%$ \\
\hline & \multicolumn{2}{|l|}{ Termo } & 90 & $38,3 \%$ \\
\hline & \multicolumn{2}{|l|}{ Pós-termo } & 0 & $0,0 \%$ \\
\hline & \multicolumn{2}{|c|}{ Não informado } & 5 & $2,1 \%$ \\
\hline \multirow{5}{*}{ Peso ao nascer (g) } & \multicolumn{2}{|l|}{ Baixo } & 41 & $17,4 \%$ \\
\hline & \multicolumn{2}{|l|}{ Muito baixo } & 36 & $15,3 \%$ \\
\hline & \multicolumn{2}{|c|}{ Extremamente baixo } & 52 & $22,1 \%$ \\
\hline & \multicolumn{2}{|l|}{$>2500 \mathrm{~g}$} & 101 & $43,0 \%$ \\
\hline & \multicolumn{2}{|c|}{ Não informado } & 5 & $2,1 \%$ \\
\hline \multirow{3}{*}{$\begin{array}{l}\text { Desfecho da } \\
\text { internação }\end{array}$} & \multicolumn{2}{|c|}{ Alta para enfermeira } & 202 & $86.0 \%$ \\
\hline & \multicolumn{2}{|l|}{ Óbito } & 5 & $2,1 \%$ \\
\hline & \multicolumn{2}{|c|}{ Transferência } & 28 & $11,9 \%$ \\
\hline
\end{tabular}

Fonte: Elaborado pelos autores (2019)

Legenda: Neonatal precoce (0 a 6 dias), neonatal tardio (7 a 27 dias), pós-neonatal (28 a 364 dias): pré-termo (<37 semanas), termo (37 a <42 semanas), pós-termo ( $\geq 42$ semanas); prematuro extremo ( $<28$ semanas), muito prematuro ( 28 a $<32$ semanas), prematuro moderado ou tardio (32 a <37 semanas); baixo peso ao nascer (<2500 g), peso muito baixo ao nascer (<1500 g), peso extremamente baixo ao nascer (<1000 g).

Tabela 2 Procedência materna de neonatos internados em Unidade de Terapia Intensiva Neonatal, Hospital Infantil, Campo Largo, Paraná, 2018.

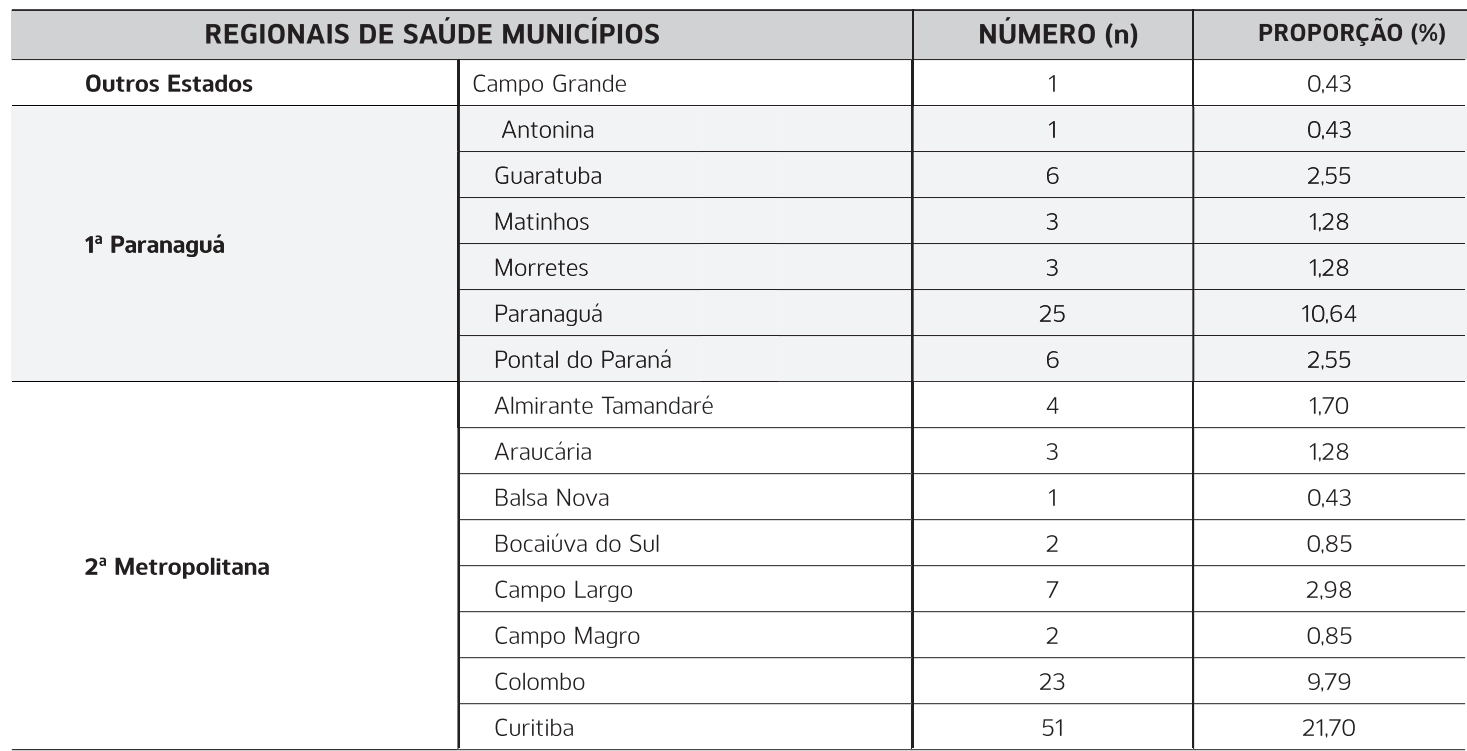




\begin{tabular}{|c|c|c|c|}
\hline \multicolumn{2}{|c|}{ REGIONAIS DE SAÚDE MUNICIPIOS } & \multirow{2}{*}{$\begin{array}{c}\text { NÚMERO (n) } \\
6\end{array}$} & \multirow{2}{*}{$\begin{array}{c}\text { PROPORÇÃO (\%) } \\
2,55 \\
\end{array}$} \\
\hline \multirow{7}{*}{$2^{\mathrm{a}}$ Metropolitana } & Fazenda Rio Grande & & \\
\hline & Itaperuçu & 2 & 0,85 \\
\hline & Lapa & 1 & 0,43 \\
\hline & Pinhais & 8 & 3,40 \\
\hline & Piraquara & 7 & 2,98 \\
\hline & São José dos Pinhais & 21 & 8,94 \\
\hline & Tunas do Paraná & 1 & 0,43 \\
\hline \multirow{6}{*}{$3^{a}$ Ponta Grossa } & Arapoti & 1 & 0,43 \\
\hline & Carambeí & 1 & 0,43 \\
\hline & Castro & 5 & 2,13 \\
\hline & Piraí do Sul & 1 & 0,43 \\
\hline & Ponta Grossa & 2 & 0,85 \\
\hline & Sengés & 1 & 0,43 \\
\hline \multirow{3}{*}{$4^{a}$ Irati } & Fernandes Pinheiro & 1 & 0,43 \\
\hline & Imbituva & 2 & 0,85 \\
\hline & Rio Azul & 2 & 0,85 \\
\hline \multirow{8}{*}{$5^{\mathrm{a}}$ Guarapuava } & Candói & 1 & 0,43 \\
\hline & Guarapuava & 1 & 0,43 \\
\hline & Laranjeiras do Sul & 1 & 0,43 \\
\hline & Marquinho & 1 & 0,43 \\
\hline & Nova Laranjeiras & 1 & 0,43 \\
\hline & Pitanga & 1 & 0,43 \\
\hline & Prudentópolis & 2 & 0,85 \\
\hline & Reserva & 1 & 0,43 \\
\hline $6^{a}$ União da Vitória & Bituruna & 1 & 0,43 \\
\hline \multirow{2}{*}{$7^{\mathrm{a}}$ Pato Branco } & Palmas & 2 & 0,85 \\
\hline & Pato Branco & 1 & 0,43 \\
\hline \multirow{3}{*}{$8^{\mathrm{a}}$ Francisco Beltrão } & Dois Vizinhos & 1 & 0,43 \\
\hline & Marmeleiro & 2 & 0,85 \\
\hline & Renascença & 1 & 0,43 \\
\hline 11ª Campo Mourão & Juranda & 1 & 0,43 \\
\hline \multirow{4}{*}{$14^{\mathrm{a}}$ Paranavai } & Alto Paraná & 1 & 0,43 \\
\hline & Loanda & 1 & 0,43 \\
\hline & Nova Londrina & 2 & 0,85 \\
\hline & Paranavaí & 1 & 0,43 \\
\hline $15^{\mathrm{a}}$ Maringá & Astorga & 1 & 0,43 \\
\hline \multirow{2}{*}{ 16 Apucarana } & Apucarana & 2 & 0,85 \\
\hline & Arapongas & 3 & 1,28 \\
\hline $18^{\mathrm{a}}$ Cornélio Procópio & São Sebastião da Amoreira & 1 & 0,43 \\
\hline $20^{\mathrm{a}}$ Toledo & Toledo & 1 & 0,43 \\
\hline \multirow{3}{*}{ 21 ${ }^{\mathrm{a}}$ Telêmaco Borba } & Ortigueira & 1 & 0,43 \\
\hline & Telêmaco Borba & 2 & 0,85 \\
\hline & Tibagi & 1 & 0,43 \\
\hline
\end{tabular}

Fonte: Elaborado pelos autores (2019) 
Em 2018, foram registrados 996 diagnósticos médicos de internação dos neonatos na UTI em estudo, distribuídos em 18 Capítulos da CID-10 e em 130 categorias diferentes. Como mostra a Tabela 3. os Capítulos quantitativamente representativos foram: XVI - Algumas afecções originadas no período perinatal e, XVII - Malformações congênitas, deformidades e anomalias cromossômicas. As categorias recorrentes foram: P07 - Transtornos relacionados com a gestação de curta duração e peso ao nascer não classificados em outra parte (13,15\%); P22 - Desconforto respiratório do recém-nascido (6.83\%); P59 - Icterícia neonatal devido a outras causas e às não especificadas (6,53\%); A41 - Outras septicemias (5,82\%); e P27 - Doença respiratória crônica originada no período perinatal (3,71\%).

Tabela 3 Diagnósticos de internação dos neonatos, Unidade de Terapia Intensiva, Hospital Infantil, Campo Largo, Paraná, 2018. *Proporção total dentre os 18 Capítulos nos quais foram classificados os Diagnósticos Médicos.

\begin{tabular}{|c|c|c|c|c|}
\hline \multicolumn{2}{|c|}{ DIAGNÓSTICOS DE INTERNAÇÃO - CID-10 } & \multirow{2}{*}{$\begin{array}{l}\text { NÚMERO } \\
\text { ABSOLUTO (N) }\end{array}$} & \multirow{2}{*}{$\begin{array}{l}\text { PROPORÇÃO NO } \\
\text { CAPITULO (\%) }\end{array}$} & \multirow{2}{*}{ TOTAL* (\%) } \\
\hline Capitulos & Categorias & & & \\
\hline \multirow{2}{*}{$\begin{array}{l}\text { Capítulo I - Algumas } \\
\text { doenças infecciosas e } \\
\text { parasitárias }\end{array}$} & A41 - Outras septicemias & 58 & 80,56 & 5,82 \\
\hline & A50 - Sífilis congênita & 7 & 9,72 & 0,70 \\
\hline \multirow{5}{*}{$\begin{array}{l}\text { Capítulo XVI - Algumas } \\
\text { afecções originadas no } \\
\text { período perinatal }\end{array}$} & $\begin{array}{l}\text { P05 - Crescimento fetal retardado e } \\
\text { desnutrição fetal }\end{array}$ & 23 & 4,16 & 2,31 \\
\hline & $\begin{array}{l}\text { P07 - Transtornos relacionados com } \\
\text { a gestação de curta duração e peso } \\
\text { ao nascer não classificados em outra } \\
\text { parte }\end{array}$ & 131 & 23,69 & 13,15 \\
\hline & $\begin{array}{l}\text { P22 - Desconforto respiratório do } \\
\text { recém-nascido }\end{array}$ & 68 & 12,30 & 6,83 \\
\hline & $\begin{array}{l}\text { P27 - Doença respiratória crônica } \\
\text { originada no período perinatal, } \\
\text { número absoluto }\end{array}$ & 37 & 6,69 & 3.71 \\
\hline & $\begin{array}{l}\text { P59 - Icterícia neonatal devido a } \\
\text { outras causas e às não especificadas }\end{array}$ & 65 & 11,75 & 6,53 \\
\hline \multirow{3}{*}{$\begin{array}{l}\text { Capítulo XVII - } \\
\text { Malformações } \\
\text { congênitas, } \\
\text { deformidades } \\
\text { e anomalias } \\
\text { cromossômicas }\end{array}$} & Q03 - Hidrocefalia congênita & 10 & 8,20 & 1,00 \\
\hline & $\begin{array}{l}\text { Q21 - Malformações congênitas dos } \\
\text { septos cardíacos }\end{array}$ & 30 & 24,59 & 3,01 \\
\hline & $\begin{array}{l}\text { Q25 - Malformações congênitas das } \\
\text { grandes artérias }\end{array}$ & 34 & 27,87 & 3,41 \\
\hline
\end{tabular}

*Proporção total dentre os 18 Capítulos nos quais foram classificados os Diagnósticos Médicos. Fonte: Elaborado pelos autores (2019)

\section{DISCUSSÃO}

No presente estudo, observou-se discreta prevalência do sexo masculino (55,7\%), concordando com dados nacionais do Censo Demográfico do Instituto Brasileiro de Geografia e Estatística (IBGE) de 2010, que apontam predomínio do sexo masculino na distribuição da população de 0 a 4 anos $^{9}$. Todavia, quando não considerada a faixa etária, há predomínio do sexo feminino $(51,03 \%)^{10}$. Achado que corrobora com o estudo de Tavares et al. (2014), desenvolvido em Minas Gerais, que 
evidenciou leve predomínio do sexo masculino $(55,8 \%)^{11}$. Também observado por Martins et al. (2013), no Rio Grande do Sul, que teve por objetivo caracterizar RN de baixo peso internados em uma UTIN dos quais 50,6\% eram do sexo masculino ${ }^{12}$.

Quanto ao tipo de parto, em desacordo ao resultado encontrado, Martins et al. (2013) demonstraram altas taxas de parto cesáreo (71,6\%), uma intervenção que deveria ser utilizada apenas em situações com indicações precisas ${ }^{12}$. A pesquisa Nascer no Brasil, um inquérito nacional sobre parto e nascimento, mostrou que o número de nascimentos por cesariana está mais de três vezes acima do recomendado pela OMS, 52,0\%, quando a taxa aceitável é de 15,0\%, expondo desnecessariamente as mulheres e os $\mathrm{RN}$ aos riscos de efeitos adversos no parto e nascimento. As altas taxas de cesariana eletiva podem estar associadas ao nascimento prematuro (pela possibilidade de erro de cálculo da idade gestacional), e ao fato de o Brasil estar entre os 10 países com maior taxa de nascimento prematuro ${ }^{13.1}$.

Em relação à condição de nascimento, Tavares et al. (2014) verificaram que a idade gestacional mínima foi de 24 semanas e a máxima de 42 semanas, que $73,7 \%$ nasceram pré-termo, baixo peso ao nascimento em $67,9 \%$ das crianças, e os pesos mínimo e máximo encontrados foram de $535 \mathrm{~g}$ e $4.745 \mathrm{~g}$, respectivamente. Em estudo realizado por Martins et al. (2013), constatou-se que 88,3\% eram prematuros, e 70,4\% apresentavam baixo peso ao nascer ${ }^{11-12}$, porcentagens significativamente maiores as observadas neste estudo, que também foram expressivas. 0 baixo peso é uma expressão da prematuridade que representa um importante fator de risco para a morbimortalidade neonatal e infantil.

Atualmente o período neonatal precoce (0-6 dias de vida) é o principal componente da $\mathrm{MI}^{14}$. correspondendo ao maior número de internações do estudo. Tavares et al. (2014) demonstraram que $43,5 \%$ das crianças permaneceram de um a 30 dias internadas, e vale ressaltar que o tempo de internação está intimamente relacionado ao diagnóstico do neonato ${ }^{11}$. O desfecho prevalente foi a alta para enfermaria, considerando-a como uma adequação entre a capacidade instalada das unidades e a condição clínica do RN, garantindo a progressão do cuidado ${ }^{15}$.

No Brasil observa-se uma cobertura crescente da assistência pré-natal,que alcança um percentual de 98\%. Entretanto, sua adequação é ainda baixa, com início tardio, número insuficiente de consultas e não adesão. Com isso, os índices de mortalidade materno-infantil ainda são preocupantes, para tanto, uma atenção pré-natal de qualidade é capaz de diminuí-los, através da detecção e intervenção antecipada de diversas situações de risco da mãe e do bebê, regulação de leitos e assistência qualificada ${ }^{16}$.

Um dos 17 Objetivos de Desenvolvimento Sustentável é assegurar uma vida saudável e promover o bem-estar para todos, em todas as idades. Dentre as metas, até 2030, acabar com as mortes evitáveis de $\mathrm{RN}$ e crianças menores de cinco anos ${ }^{17}$. Apesar do declínio, que tem se mantido constante, o relatório Levels and Trends in Child Mortality 2017 mostrou que em 2016 houve 15 mil crianças mortas, destas, $46,0 \%$ nos primeiros 28 dias de vida ${ }^{18}$.

Mais da metade das internações descritas no estudo (58,74\%) foram associadas à prematuridade (P07), que se constitui em maior fator de risco para morbidade e morte do RN. A pesquisa Nascer no Brasil revelou que a taxa de prematuridade brasileira (11,5\%) é quase duas vezes superior à observada nos países europeus. Quanto à sua ocorrência, a prematuridade espontânea ocorreu em 59,0\%; por indução do parto ou cesariana anteparto foi de 41,0\%; e cesariana sem trabalho de parto foi de $90,0 \%{ }^{19}$. Dentre os desfechos negativos observados a esta população, citam-se: uso de oxigênio; internação em UTIN; hipoglicemia; problemas respiratórios; icterícia; e atraso no aleitamento materno ${ }^{20}$.

A OMS traz que $75,0 \%$ dos prematuros 
podem sobreviver com cuidados essenciais (para o binômio mãe e bebê) que nem sempre trazem um custo elevado ao serviço, como: a prevenção de hipotermia; o apoio ao aleitamento materno; a prevenção ou tratamento de infecções perinatais; e o Método Canguru. Ainda existe a prevenção de mortes por complicações da prematuridade que inicia na gestação, com um pré-natal de qualidade, incentivo à alimentação saudável e à prevenção do uso de drogas antes e durante a gestação, aplicando-se a identificação dos riscos com atenção centrada na prevenção das infecções'

\section{CONSIDERAÇÕES FINAIS}

A caracterização das internações de uma Unidade de Terapia Intensiva Neonatal reforça o perfil encontrado em outros estudos e a necessidade de uma assistência de alta qualidade para a manutenção da redução da mortalidade neonatal e infantil. Os RN com maior risco de morte e incapacidade, assistidos nesta unidade, foram aqueles com complicações relacionadas à prematuridade, reiterando a importância de investimentos na assistência pré-natal, a fim de reduzir a incidência de partos prematuros e suas complicações e a importância do cuidado especializado, por profissionais de saúde com qualificação, a fim de evitar as mortes por causas evitáveis. Propõem-se novas pesquisas voltadas para a qualidade de vida de neonatos com projeção para a sobrevida das crianças e seu desenvolvimento saudável.

\section{REFERÊNCIAS}

1. World Health Organization. Preterm birth [Internet]. Geneva: WHO: 2018 [citado em 2019 Fev 20]. Disponivel em: https://www. who.int/news-room/fact-sheets/detail/preterm-birth.

2. Ribeiro JF, Silva LLC, Santos IL, Luz VLES, Coêlho DMM. O prematuro em unidade de terapia intensiva neonatal: a assistência do enfermeiro. The premature newborn in neonatal intensive care unit: the nurse's care. Rev Enferm UFPE on line. 2016:10(10):383341.

3. Oliveira MG, Viau AC, Heidemann LA, Nicoloso L, Volkmer DFV Mortalidade neonatal precoce em recém-nascidos de muito baixo peso: estudo de coorte. Bol Cient Ped. 2015:4(3):75-9.

4. Careti CM, Scarpelini AHP, Furtado MCC. Perfil da mortalidade infantil a partir da investigação de óbitos. Rev Eletrônica Enferm. 2014:16(2):352-60.

5. Brasil, Ministério da Saúde. Portaria $n^{\circ} 371$, de 7 de maio de 2014. Institui diretrizes para a organização da atenção integral e humanizada ao recém-nascido (RN) no Sistema Único de Saúde (SUS) [Internet]. Saúde Legis - Sistema de Legislação da Saúde. 2014 [citado em 2019 Fev 10]. Disponivel em: http://bvsms.saude. gov.br/bvs/saudelegis/sas/2014/prt0371_07_05_2014.html.

6. Paraná. Secretaria de Estado da Saúde. Hospital Infantil Waldemar Monastier [Internet]. Campo Largo: Hospital Infantil Waldemar Monastier; [citado em 2019 Ago 30]. Disponível em: http://www.hospitalinfantil.saude.pr.gov.br/.

7. Correia LOS, Padilha BM, Vasconcelos SML. Métodos para avaliar a completitude dos dados dos sistemas de informação em saúde do Brasil: uma revisão sistemática. Ciên Saúde Colet. 2014:19(11):4467-78.

8. Cuschieri S The STROBE guidelines. Saudi J Anaesth [Internet] 2019 Apr [citado 2019 Out 10]:13(Suppl 1):S31-S34. Disponivel em: http://www.saudija.org/text.asp?2019/13/5/31/252631. doi: 10.4103/sja. SJA_543_18 PMCID: PMC6398292.

9. Instituto Brasileiro de Geografia e Estatística (IBGE) Distribuição da População por sexo segundo os grupos de idade Brasil - 2010 [Internet]. Brasilia: IBGE; c2019 [citado em 2019 Jun 18]. Disponivel em: https://brasilemsintese.ibge.gov.br/populacao/ populacao-por-sexo-e-grupo-de-idade-2010.html.

10. Instituto Brasileiro de Geografia e Estatística (IBGE) Distribuição da População por sexo segundo Unidades da Federação - Brasil - 2010 [Internet]. Brasília: IBGE; c2019 [citado em 2019 Jun 18]. Disponivel em: https://brasilemsintese.ibge. gov.br/populacao/populacao-por-sexo-segundo-as-unidades-dafederacao.html.

11. Tavares TS, Duarte ED, Silva BCN, Paula CM, Queiroz MPM, Sena RR. Caracterização do perfil das crianças egressas de unidade neonatal com condição crônica. Rev Enferm Cent.- Oeste Min [Internet]. 2014 [citado em 2019 Out 10];4(3):1322-35. Disponivel em: http://www.seer.ufsj.edu.br/index.php/recom/ article/view/802.

12. Martins EL, Padoin SMM, Rodrigues AP, Zuge SS, Paula CC Trojahn TC, Bick MA. Caracterização de recém-nascidos de baixo peso internados em uma unidade de terapia intensiva neonatal. Rev Enferm UFSM. 2013:3(1):96. 
13. Universidade Federal do Piaui. Nascer no Brasil: resultados da maior pesquisa sobre parto e nascimentos [Internet]. Teresina: UFPI; 2014 [citado em 2019 Jun 18]. Disponivel em: https://www.ufpi. br/ultimas-noticias-ufpi/5864-nascer-no-brasil--resultados-\%20 $\% 20$ da-maior-pesquisa-sobre-parto-e-nascimentos.

14. Lansky S, Friche AAL, Silva AAM, Campos D, Bittencourt SDA, Carvalho ML, et al. Pesquisa Nascer no Brasil: perfil da mortalidade neonatal e avaliação da assistência à gestante e ao recém-nascido. Cad Saúde Pública. 2014:30(suppl 1):S192-207.

15. Brasil, Ministério da Saúde. Portaria n 930, de 10 de maio de 2012. Define as diretrizes e objetivos para a organização da atenção integral e humanizada ao recém-nascido grave ou potencialmente grave e os critérios de classificação e habilitação de leitos de Unidade Neonatal no âmbito do Sistema Único de Saúde (SUS) [Internet]. Saúde Legis - Sistema de Legislação da Saúde. 2012 [citado em 2019 Fev 2010]. Disponivel em: http://bvsms.saude. gov.br/bvs/saudelegis/gm/2012/prt0930_10_05_2012.html.

16. Santana TCP, Silva LM, Silva LRFG, Rocha LM, Canhoto CTS, Silva ACFA, et al. Dificuldades dos enfermeiros no atendimento ao pré-natal de risco habitual e seu impacto no indicador de morbimortalidade materno-neonatal. Rev Eletrônica Acervo Saúde. 2019:(20):e711.

17. Organização das Nações Unidas. Transformando nosso Mundo: a agenda 2030 para o desenvolvimento sustentável [Internet]. Rio de Janeiro: UNIC Rio, 2015 [citado em 2019 Jun 18]. Disponível em: https://nacoesunidas.org/pos2015/agenda2030/

18. Organização Pan-Americana de Saúde Apesar do declínio constante na mortalidade entre crianças com menos de cinco anos, 7 mil recém-nascidos morrem todos os dias, afirma novo relatório [Internet]. Brasília: OPAS, 2017 Out 19 [citado em 2019 Jun 18]. Disponível em: https://www.paho.org/bra/index. php?option=com_content \&view=article\&id=5530:apesar - do declínio-constante-na-mortalidade-entre-criancas-com-menosde-cinco-anos-7-mil-recem-nascidos-morrem-todos-os-diasafirma-novo-relatorio\&ltemid $=820$

19. Leal MC. Childbirth and birth in Brazil: an evolving scenario. Cad Saúde Pública. 2018:34(5):1-3.

20. Fundação Oswaldo Cruz. Taxa de bebês prematuros no país é quase o dobro do que em países da Europa [Internet]. Rio de Janeiro: FIOCRUZ, 2016 Dez 7 [citado em 2019 Jun 18]. Disponível em: https://portal.fiocruz.br/noticia/taxa-de-bebes-prematurosno-pais-e-quase-o-dobro-do-que-em-paises-da-europa. 